\title{
A Self-powered Biosensor with a Flake Electrochromic Display for Electrochemical and Colorimetric Formaldehyde Detection
}

Xiaoxuan Sun, ${ }^{\dagger, \ddagger}$ He Zhang, ${ }^{\dagger, \ddagger}$ Shuai Hao, ${ }^{\dagger, \ddagger}$ Junfeng Zhai, ${ }^{*, \dagger}$ Shaojun Dong ${ }^{*,+, \ddagger}$

†State Key Laboratory of Electroanalytical Chemistry, Changchun Institute of Applied Chemistry, Chinese Academy of Sciences, Changchun 130022, China

‡University of Science and Technology of China, Hefei 230026, China 


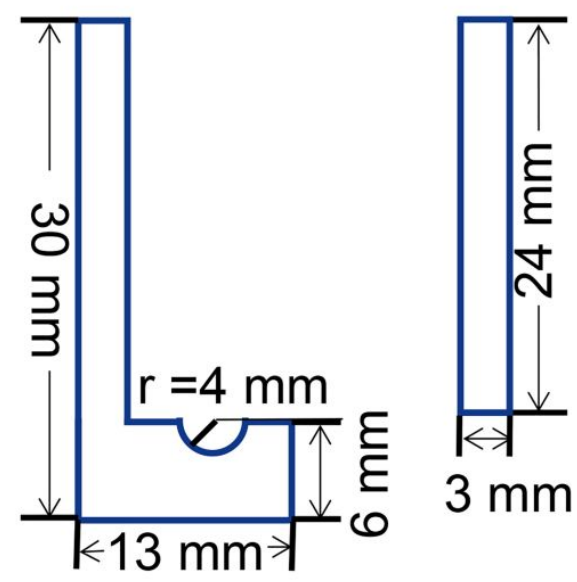

Figure S1. The size and shape of the ITO-PET. 


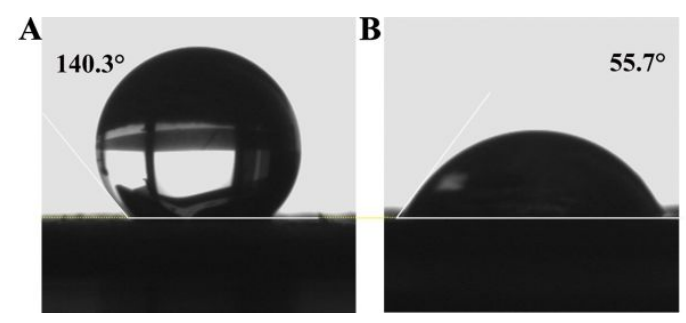

Figure S2. The image of the contact angel of a drop of water on primary BP (A) and BP surfaces (B). 


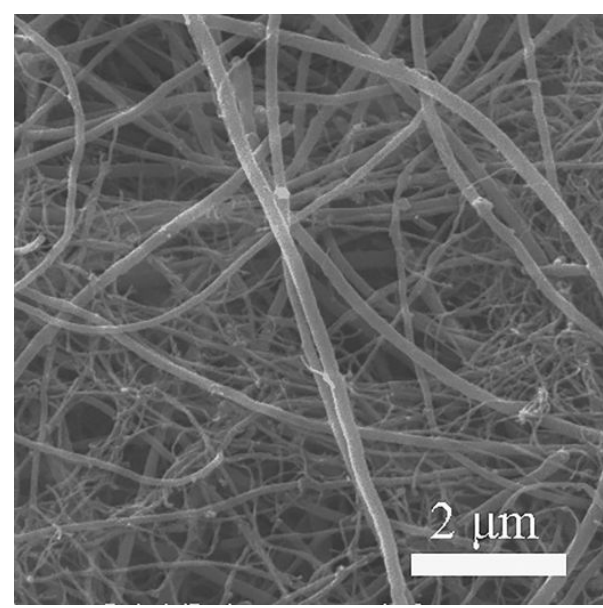

Figure S3. A SEM image of the BP electrode. 


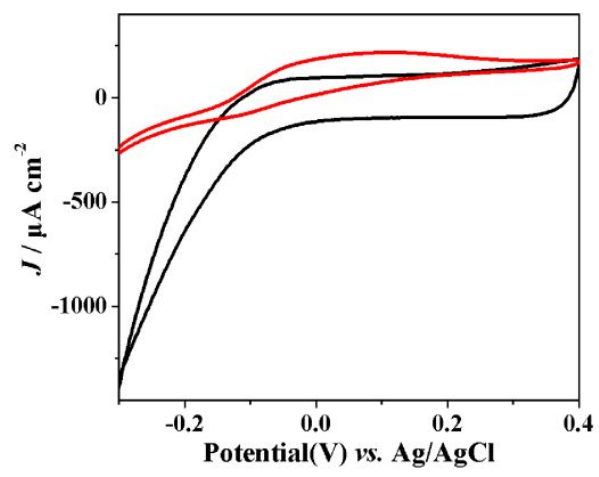

Figure S4. CVs of the BP electrode in 0.1 M PBS buffer, $\mathrm{pH}$ 6.0, with (red) and without (black) $5 \mathrm{mM} \mathrm{NADH}$ at a scan rate of $5 \mathrm{mV} \mathrm{s}^{-1}$. 


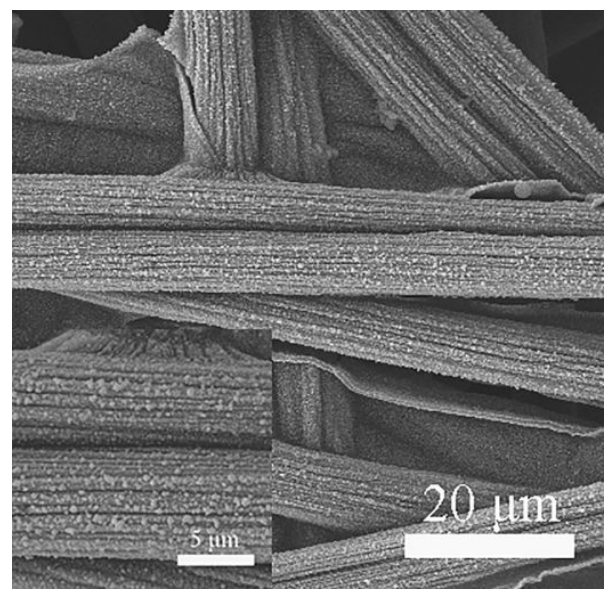

Figure S5. A SEM image of the Au NPs/CFP electrode (inset: SEM image). 


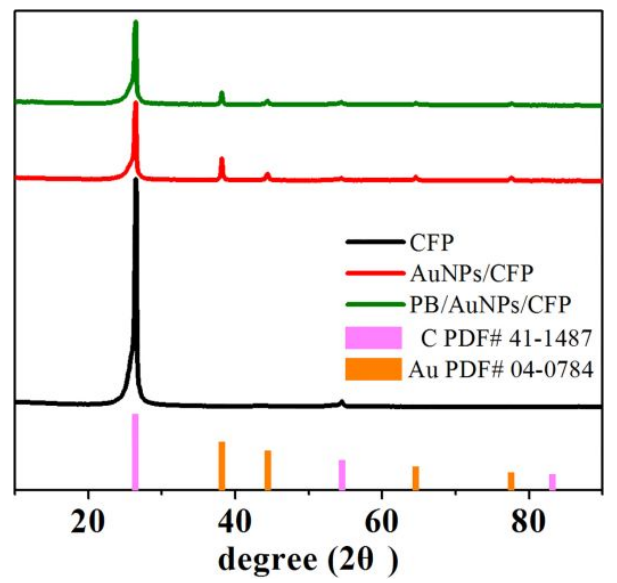

Figure S6. XRD patterns of CFP, Au NPs/CFP and PB/Au NPs/CFP electrodes. 

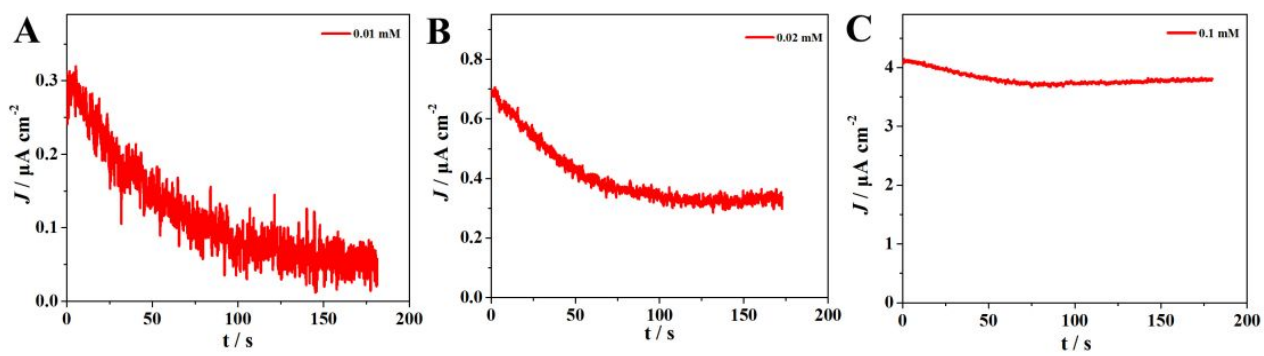

Figure S7. Chronoamperometric responses of ESPB to formaldehyde with the concentrations from $0.01 \mathrm{mM}(\mathrm{A}) 0.02 \mathrm{mM}(\mathrm{B})$ and $0.1 \mathrm{mM}(\mathrm{C})$. 


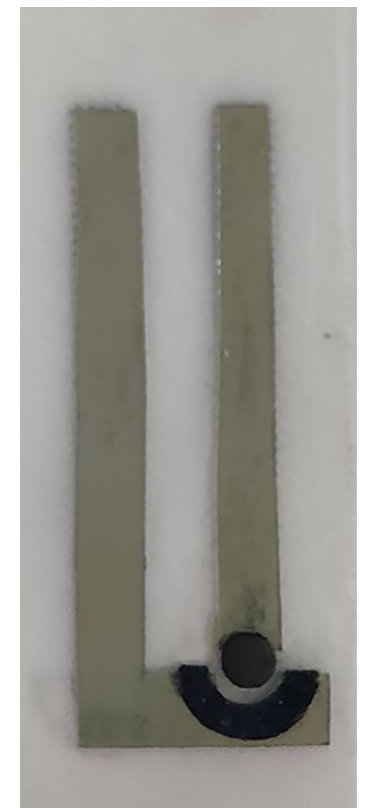

Figure S8. A photograph of the ESPB. 

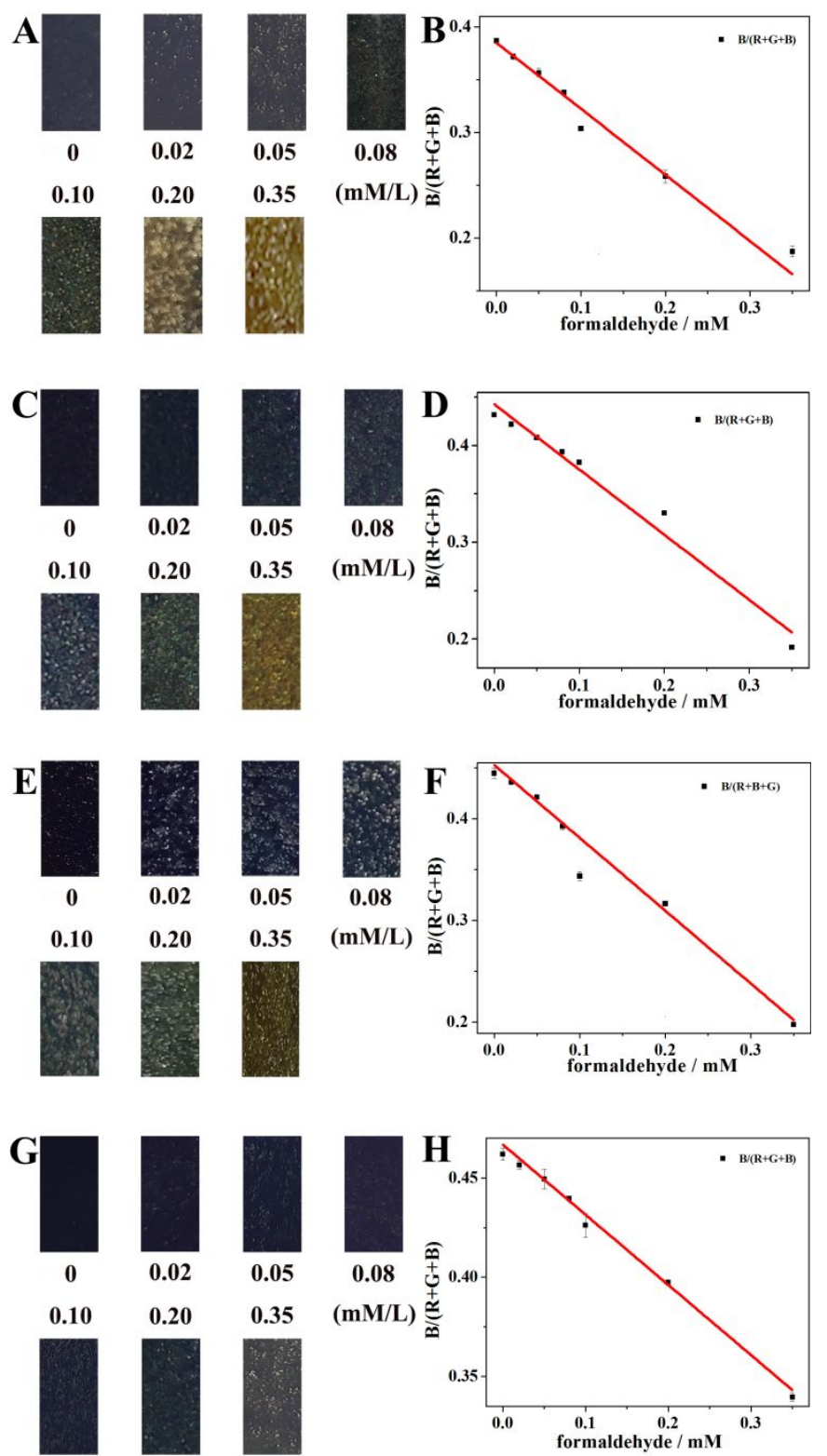

\begin{tabular}{|c|c|c|}
\hline PB amount & Slope $\left(\mathrm{L} \mathrm{mM}^{-1}\right)$ & $\mathrm{R}^{2}$ \\
\hline $3 \mathrm{~min}$ & -0.62 & -0.96 \\
\hline $4 \mathrm{~min}$ & -0.67 & -0.97 \\
\hline $5 \mathrm{~min}$ & -0.72 & -0.98 \\
\hline $6 \mathrm{~min}$ & -0.35 & -0.99 \\
\hline
\end{tabular}

Figure S9. Colorimetric cards and calibration curves obtained with the ESPB composed of the cathodes loading different amount of PB (A-H). The cathodes are prepared by immersing $\mathrm{Au} \mathrm{NPs} / \mathrm{CFP}$ in a solution containing $\mathrm{K}_{3}\left[\mathrm{Fe}(\mathrm{CN})_{6}\right]$ and $\mathrm{FeCl}_{3}$ for $(A, B) 3$ minutes, $(C, D) 4$ minutes, $(E, F) 5$ minutes, $(G, H) 6$ minutes, each point is the average of three test. (I) Comparison of optical sensitivity of ESPB with cathode of different PB amount. 